\title{
A New Tensile Steel Strip Cable Dome and A Force-finding Method of Approaching Initial Shape
}

\author{
GUAN Dongzhi ${ }^{1, a}$, ZHU Mingliang ${ }^{1, b}$, JIANG Huanzhi ${ }^{1, c^{*}}$, \\ CHEN Xueqi ${ }^{1, d}$ and GUO Zhengxing ${ }^{1, e}$ \\ ${ }^{1}$ School of Civil Engineering, Southeast University, Nanjing, China \\ a gdzh.js@163.com, b zhumingliangseu@163.com, c704358537@qq.com \\ d704358537@qq.com, e'guozx1956@126.com
}

Keywords: cable dome, tensile steel strip, force finding

Abstract. Cable domes has been widely adopted around the world. A new tensile steel strip cable dome is proposed and a force-finding method of approaching initial shape is introduced. It is easy to be constructed by tow-lifting method without many scaffolds and connect rigid materials directly. The force-finding method of approaching initial shape is effective for the proposed structure.

\section{Introduction}

The cable dome system was proposed by Fuller in his patent of 1962 in the form of a triangular cable with compressive struts hinging on it [1]. Geiger designed the first cable dome for the Seoul Olympics gymnastics and fencing halls in 1986 [2]. After that, cable domes have been widely adopted in many countries as a basic form of large-span structures. The largest cable dome is the one called Georgia dome in the Atlanta Olympics [3].

An ordinary cable dome consists of ridge cables, oblique cables, ring cables and struts. Without an initial prestress, the cables are flexible and the cable domes cannot carry any external loads. In other words, the initial prestress in the cables and struts make the cable dome rigid enough to become a structure resisting loads [4]. There are two classes of roofing materials for cable domes, they are flexible roofs like tensioned membranes and rigid roofs like thin steel plates or glass. Currently, most of cable domes are covered by the flexible roofing materials, which are tensioned membranes.

It is advantageous for tensioned membranes to be lightweight and have the ability to span a long distance with appropriate prestress. However, there are some shortcomings hindering membranes to be applied extensively, such as high cost, large deformation, low bearing capacity, poor insulation performance and poor anti-pollution performance.

In terms of rigid roofing materials like glass and steel plates, the above-mentioned weakness of membranes can be overcome by their inherent properties. And cable domes are increasingly used with the help of rigid roofing materials especially in China [5].

For the characteristics of cables and rigid roofs, they cannot be connected together directly. In most cases, substructures are constructed to support rigid roofs and connect cables. Moreover, cable clamp of complex configurations are needed to support substructures on cable domes. In Wuxi New District Science \& Technology Communication Center, a single-layer reticulated shell was put on the top of struts in a cable dome [6]. In Taiyuan Coal Trading Center, an orthogonal single-layer cable net was constructed on the ridge cables to support a glass roof [7].

This paper aims at proposing a new structure called tensile steel strip cable dome to facilitate the combination of cable dome structure and rigid roofs. It has not only the advantages of traditional cable domes like lightweight and long-span capacity, but also the characteristic that it is mechanism system when not tensioned. Thus, it can be constructed without many scaffolds like traditional cable domes, and the steel strips make it easy to build rigid roofs on the bearing structures by removing substructures. A simple and effective 
force-finding method is introduced and applied to find the force distribution of the tensile steel strip cable dome.

\section{Tensile steel strip cable dome}

Structure characteristics. The tensile steel strip cable domes are created by replacing the ridge cables of traditional cable domes into narrow and thin steel plate. Similar to traditional cable domes, the tensile steel strip cable domes consist of rigid ring, oblique cables, struts, ring cables, and tensile steel strips (shown in Fig. 1). The tensile steel strips are made of long hot-rolled steel plates with relative high tensile strength. As the little thickness of the tensile steel strips relative to the long span, they are flexible without much stiffness like ordinary cables when not tensioned. Therefore, advanced construction method of cable domes like tow-lifting construction technology can be used to the new structure [8]. After the tensile steel strip cable dome is tensioned, the prestress distribution generates and the mechanism system become a rigid structure to bear external loads. Then the rigid roof can be installed on the tensioned steel strips by the high strength bolts and nuts. The holes for the bolts are opened when the steel strips are produced.

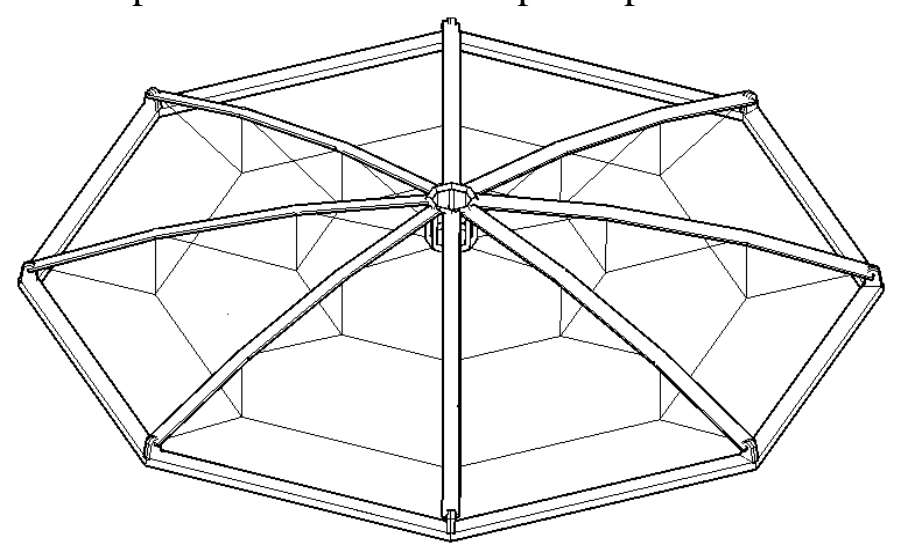

Fig. 1 Components of tensile steel strip cable dome

To meet the real structural demand of tensile strength, several same steel strips are overlapped up and down and fixed together by high-strength bolts to act as one component. The connections between the tensile steel strips, struts and oblique cables are very easy to make just with some steel cover plates, joint plates and high-strength bolts. As shown in Fig. 2, the strut and oblique cable are connected to the joint plate through the hinge pin, and the steel cover plate is located on the tensile steel strip. The high-strength bolts fix the cover plate, steel strip and joint plate together with adequate prestress. The installation of the connection can be conducted on the ground before lifting process. Owing to the bolt holes opened on the tensile steel strips, it is also very convenient to install the rigid roofing materials on the new structures, as shown in Fig. 3. Similar to the above-mentioned connection, the rigid roofing materials like keels are fixed onto the tensile steel strips just by high-strength bolts and nuts.
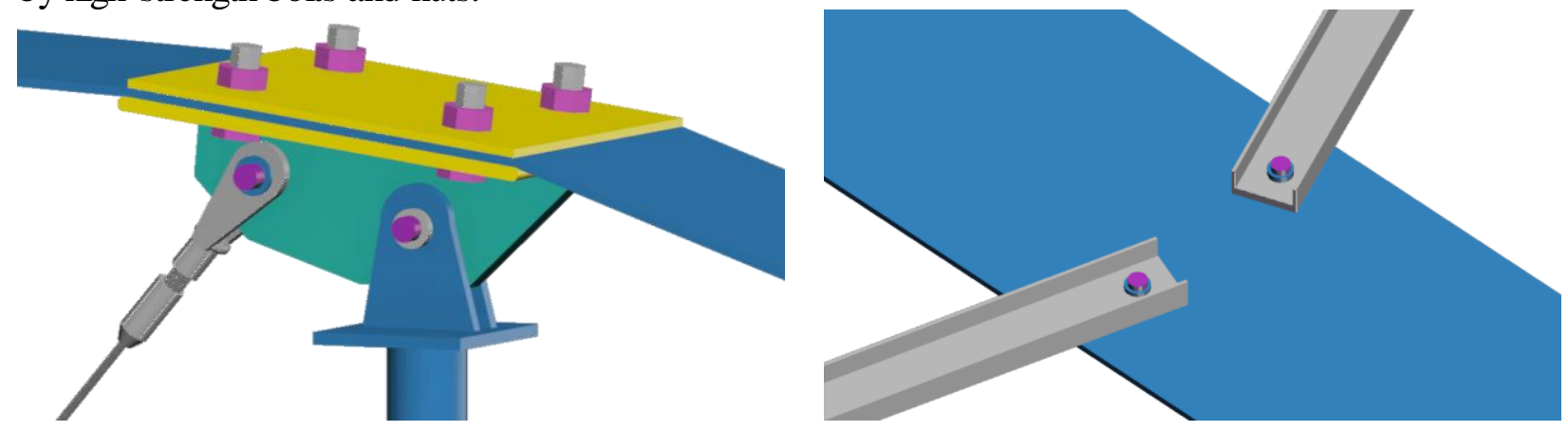

Fig. 2 Connection on struts

Fig. 3 Connection between roof materials and steel strips 


\section{The force-finding method of approaching initial shape}

Basic priciple. Because the tensile steel strip cable dome provide no stiffness to bear external loads as the traditional cable dome, pretress distribution must be found and built in the new structure. A simple force-finding method based on iteration, shown in Fig. 4, is introduced as follows: a cable of a length $L$ elongates under a force $\mathrm{F}$. The elongation $\Delta L$ can be calculated by Eq. 1 and the strain $\varepsilon_{l}$ generated can be attained by Eq. 2 (Fig. 4 b). If an initial strain $\varepsilon_{0}$ or equivalent temperature $\Delta T$, which is equal to $\varepsilon_{l}$, is applied on the cable, the cable would recover its initial length (Fig. 4c). Using the basic principle to iterate, the initial configuration of structures can be approached gradually. As a result, the prestess distribution could be known.

$$
\begin{gathered}
\Delta L=\frac{F L}{E A} . \\
\varepsilon_{l}=\frac{F}{E A} . \\
\Delta T=\frac{\varepsilon_{0}}{\alpha} .
\end{gathered}
$$

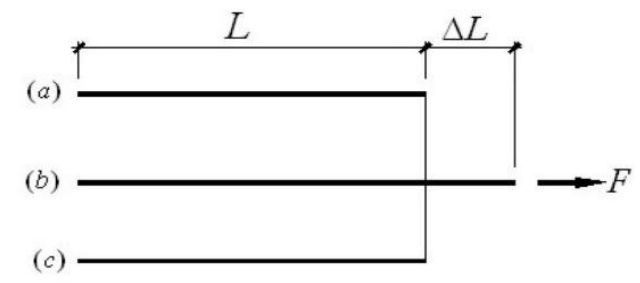

Fig. 4 Schematic of approaching initial shape method

FEM model. In order to know about the basic structural performance of the tensile steel strip cable dome, a small FEM model with a $8 \mathrm{~m}$ span is set up using the ANSYS software [9]. The hot-rolled steel plates of a section $150 \times 3 \mathrm{~mm}$ are used as tensile steel strip, steel pipes $\Phi 30 \times 2.5$ act as struts, and the high-strength bars of $\Phi 7$ become the oblique cables and ring cables. The model suffers its self-weight and concentrated load of $30 \mathrm{kN}$ on each strut. The force-finding method illustrated above is utilized to find the prestress distribution of the model. The cable force in the condition of the found initial prestress distribution and the loading is shown in Fig. 5, and the deformation under the same condition is depicted in Fig. 6. It can be seen that the model with the prestress distribution calculated by the simple method generates negligible deformation under the loading condition. Thus, the method introduced above is effective find the prestress distribution for the tensile steel strip cable dome.

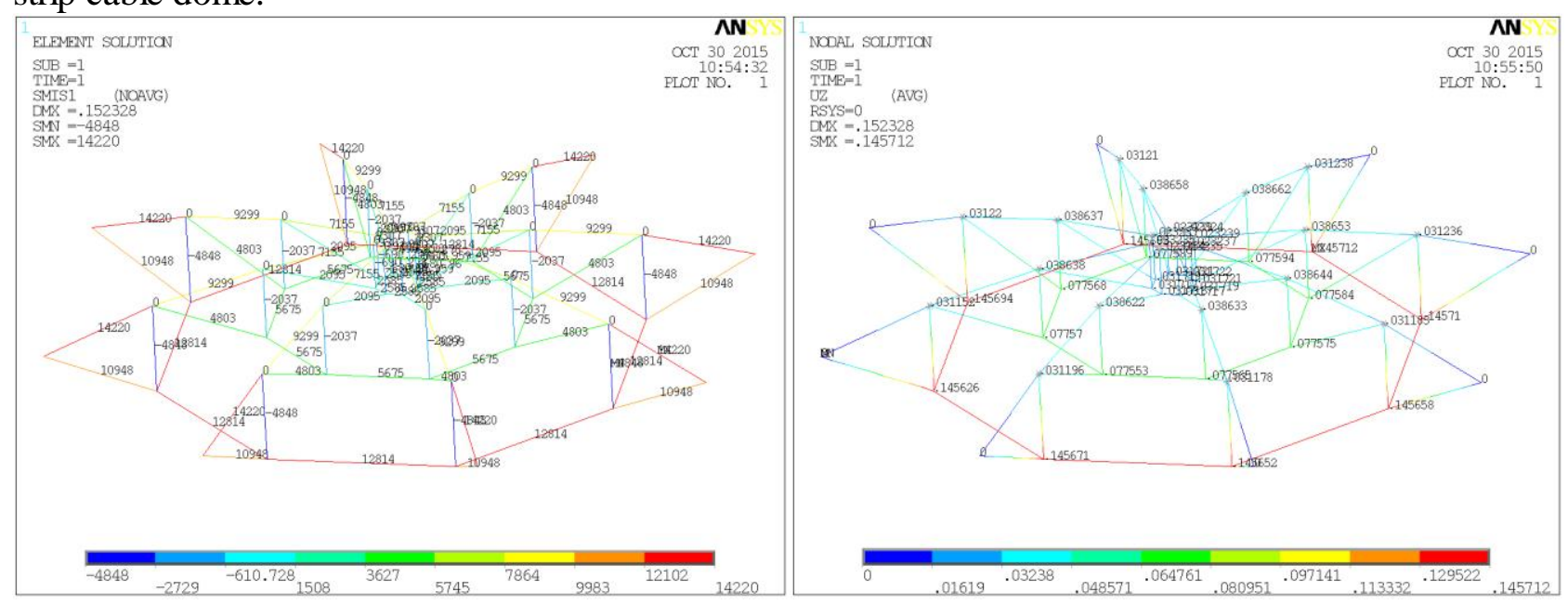

Fig. 5 Force of cables in the FEM model

Fig. 6 Results of deformation in FEM model 


\section{Summary}

As the increasing development of cable domes and finished construction of two cable domes with rigid roofing materials in China, a new tensile steel strip cable dome is proposed and a force-finding method of approaching initial shape is introduced. The tensile steel strips take the place of the ridge cables in traditional cable domes, leading to convenience of connecting rigid roofing materials with bearing system. And it can be also constructed as the traditional cable domes without many scaffolds. The force-finding method of approaching initial shape is effective for the new structure.

\section{Acknowledgements}

The authors acknowledge financial support from the Research Innovation Program for College Graduates of Jiangsu Province (Grant No. KYLX_0149) and A Project Funded by the Priority Academic Program Development of Jiangsu Higher Education Institutions (Grant No. CE02-1-15, CE01-2-5).

\section{References}

[1] Fuller, R. Buckminster. Synergetics. Pacific Tape Library, New York, 1975.

[2] Geiger, David H., Andrew Stefaniuk, and David Chen. The design and construction of two cable domes for the Korean Olympics. Proc. of the IASS Symposium on Shells, Membranes and Space Frames. 2(1986) 265-272.

[3] Levy, Matthys P. The Georgia Dome and beyond: achieving lightweight-longspan structures. Spatial, lattice and tension structures: proceedings of the IASS-ASCE International Symposium (1994) 560-562. [4] Wang, Zhenhua, Xingfei Yuan, and Shilin Dong. Simple approach for force finding analysis of circular Geiger domes with consideration of self-weight. Journal of Constructional Steel Research 66.2 (2010) 317-322.

[5] Wang, Z. H. Theoretical and Experimental Research on a New Spatial Structure Composed of Cable Dome and Single-layer Lattice Shell. Hangzhou: Zhejiang University (2009). (In Chinese)

[6] Zhengxing, Guo, et al. Key Construction Technology of Rigid Roof Cable Dome and Engineering Application. Construction Technology 8 (2010): 020. (In Chinese)

[7] Luo, Bin, Zhengxing Guo, and Feng Gao. Research on non-bracket tow-lifting construction technology and complete process analysis of cable dome. Journal of Building Structures 5 (2012): 004. (In Chinese)

[8] Guo, Z.X., Zong, Z.L., Luo, B., et al. China Patent ZL200810234362.1. (2010) (in Chinese)

[9] ANSYS version 12.0 on-line user's manual (2009) 